\title{
Channel impulse response equalization scheme based on particle swarm optimization algorithm in mode division multiplexing
}

\author{
Shaymah Yasear $^{1, *}$ and Angela Amphawan ${ }^{1,2}$ \\ ${ }^{1}$ School of Computing, Universiti Utara Malaysia, Sintok, Kedah, Malaysia \\ ${ }^{2}$ Research Laboratory of Electronics, Massachusetts Institute of Technology, Cambridge, Massachusetts, USA
}

\begin{abstract}
Mode division multiplexing (MDM) technique has been introduced as a promising solution to the rapid increase of data traffic. However, although MDM has the potential to increase transmission capacity and significantly reduce the cost and complexity of parallel systems, it also has its challenges. Along the optical fibre link, the deficient characteristics always exist. These characteristics, damage the orthogonality of the modes and lead to mode coupling, causing Inter-symbol interference (SI) which limit the capacity of MDM system. In order to mitigate the effects of mode coupling, an adaptive equalization scheme based on particle swarm optimization (PSO) algorithm has been proposed. Compared to other traditional algorithms that have been used in the equalization process on the MDM system such as least mean square (LMS) and recursive least squares (RLS) algorithms, simulation results demonstrate that the PSO algorithm has flexibility and higher convergence speed for mitigating the effects of nonlinear mode coupling.
\end{abstract}

\section{Introduction}

The unstoppable and increasing demand for data traffic requires providing higher bandwidth to end users[1]. To cope with the rapid increase of data traffic; for many years the researchers focused on the development and improve the efficiency of optical fibre transmission system [2].

The MDM transmission system is considered as a promising solution. However, in the optical MDM system, when the light ray propagates through the MMF; it spreads into multiple paths (modes). Some of these modes (called low-order modes) travel at low angles to the fibre axis. These modes have shorter paths compared to the other modes (high-order modes) which travel at larger angles to the core axis and they have longer paths. Therefore, these modes travel through the fibre with different group velocities (i.e., differential mode group delays (DMGD)), and arrive to the receiver at different times[3]. During propagation of these modes, and due to the fibre manufacturing imperfections (e.g., bending, stresses and twisting), the orthogonality of the modes will damage and the modes will couple with each other, causing the so-called mode coupling [4]. Each time the mode coupling occurs, the power is leaked from one data symbol launched into a particular mode to the adjacent symbol so-called cross-talk. This causes the symbols to spread over time. This phenomenon is known as modal dispersion (MD). The modal dispersion will lead to overlapping the neighboring symbols of signal while it transmits to the receiver. The signal is then no longer usable. This phenomenon is known as inter-symbol interference (ISI) [5]. In the optical communication, each pulse represents a symbol and each symbol or several symbols representing digital information (bit). Due to the mode coupling effects, the receiver cannot restore the transmitted symbols and recover their information. The mode coupling is responsible for the bandwidth limitation of the transmitted signal and may lead to significant increase in bit error rate (BER) in long transmission distances [6].

To mitigate the effects of mode coupling in the MDM system, several techniques have been proposed [7, 8]. However, the hardware-based equalizer cannot provide an acceptable performance with time-varying channels, due to the implementation complexity. Moreover, a simple linear equalizer is difficult to meet the basic requirements of the system. In this case, an equalizer based on an adaptive algorithm must be used.

The adaptive equalizer is implemented using algorithms that have conditions where it is periodically check and compensate or equalize the transmission channel characteristics. In addition to, restore the transmitted symbols and recover their information. However, the traditional adaptive equalization algorithms, that have been used with MDM system; such as least mean square (LMS), recursive least squares (RLS) have drawbacks such as slow convergence, speed, poor stability, high computational complexity and inaccurate equalization result $[4,9]$. Furthermore, the performance of linear equalization algorithm is degraded in channels having

\footnotetext{
*Corresponding author: shayma1985akram@gmail.com
} 
large eigenvalue spread [10]. For these reasons, the use of these algorithms has given rise to the limited performance of the optical MDM system. Therefore, it is worth investigating an alternative algorithm which is able to cope with a large eigenvalue spread of the channel. One possible solution that has been proposed to overcome these issues is using the particle swarm optimization (PSO) algorithm. The remainder of this paper is as follows. Section 2 presents MDM system, Section 3 reviews the other conventional algorithms, while Section 4 and Section 5 discusses the result of equalization schemes and the conclusion respectively.

\section{Related work}

To date, most studies realized on using an equalizer based on LMS and RLS algorithms to mitigate the effect of mode coupling in MDM system [11].

\subsection{Equalization schemes in MDM system}

In[12], LMS and constant modulus algorithm (CMA) have been proposed to be used with MDM system. Although the LMS algorithm has a low computational complexity, its training sequence adds an overhead and thus reduces the performance of the system. Moreover, the CMA requires a suitable learning step; the equalizer converges slowly with small steps and cannot converge with large steps for accurate estimation [8].In [13], Koebele et al., discuss the use of CMA to update the equalizer coefficients, although, CMA provides low computational complexity, it has a poor performance. This is due to the instability and a slower convergence rate compared with LMS algorithm[14, 15]. In [16], proposed Single-Carrier frequency domain equalization (SC-FDE), has low computational complexity; compared to time domain equalization (TDE). However, FDE it still suffers from high hardware complexity. This is due to large fast Fourier transform (FFT) size determined by the channel impulse response spread[17].In [4], the performance of adaptive FDE based on LMS and RLS algorithms has been reviewed. LMS algorithm suffers from low cyclic prefix (CP) efficiency and higher SERs compared with RLS. In the other hand, RLS, suffers from high computational complexity and high cost and power consumption. In [17], a single-stage FDE based on RLS algorithm (FD-RLS) has been proposed. According to the authors, the purposed single-stage adaptive FD-RLS algorithm provides low hardware complexity compared with the two-stage method. However, the RLS algorithm still suffers from instability and high computational complexity.

\subsection{Current PSO-based equalization schemes in wireless radio system}

The PSO algorithm has been proposed by Kennedy and Eberhart in 1995 [25], aims to seek the optimal solution, in a search space. Recently, several studies [17, 26-28] have investigated the use of PSO algorithm in adaptive equalization processes. These studies demonstrated that the PSO algorithm outperforms other traditional algorithms that have been used in the adaptive equalization of the MDM system. The PSO algorithm provides a far more efficient performance in a channel with a large eigenvalue spread compared to traditional algorithms. The convergence speed of PSO algorithm is faster than LMS algorithm. Furthermore, the PSO algorithm is able to reduce the BER and provides a high convergence rate compare to LMS and RLS algorithms. In nonlinear channel; the PSO-based algorithms outperform the LMS algorithm, especially in heavilydistorting channels. For this reason, this study will focus on investigating the possibility of improving the performance of MDM system by minimizing the effect of mode coupling by using adaptive equalization based on PSO algorithm. In[18], the PSO algorithm was used to train the functional link artificial neural networks (FLANNs) equalization, in order to enhance its classification capability. According to the authors, the PSO algorithm provides a better result compare to LMS and BP algorithm. In [19], the performance of a different versions of PSO algorithm was studied and compared with the LMS-based algorithm. The study demonstrated that, PSO algorithms converge faster than LMS algorithm. In [20], a new resource management technique for the orthogonal frequency division multiplexing (OFDM) technique was proposed based on PSO algorithm, to solve the problem of sub-channel resource allocation in downlink of OFDMA system. In their study, the authors assumed the channel is constant during allocation. With this assumption, the PSO-based technique provided better performance compared to other methods. the performance of adaptive equalizer based on LMS, RLS and PSO algorithms in wireless mobile communication was compared. The algorithms were tested with the help of three parameters, MSE, BER and time of convergence (TOC). The results of the study demonstrated that, compare to LMS and RLS algorithms, PSO algorithm is more efficient in reducing the MSE and BER. In [19], an adaptive frequencydomain (FD) equalizer for the single-carrier frequency division multiple access (SC-FDMA) system was designed based on modified PSO algorithm. $\operatorname{In}[10]$, the PSO algorithm integrated with the LMS algorithm to improve the decision feedback equalization (DFE). According to the authors, the proposed technique reduces the complexity and provide better performance compared to LMS and RLS algorithms.

The optical MDM system shares some similarities with the wireless radio MIMO system. In optical MDM system the manufacturing imperfections in the MMF lead to mode coupling, giving rise to chromatic dispersion (CD) and modal dispersion (MD). This induces multipath propagation similar to that in wireless radio MIMO systems caused by scattering and Doppler effects. The success of PSO-based equalization algorithms in wireless radio systems and the similarity in the multipath characteristics of both optical MDM and 
wireless radio MIMO systems motivates the adaptation of the PSO-based equalization algorithm for optical MDM systems.

\section{Simulation model}

\subsection{Modelling MDM system}

In order to evaluate the performance of PSO equalization scheme, a MDM system is simulated using Optisystem simulation software. Figure 1 shows the configuration of the simulated link.

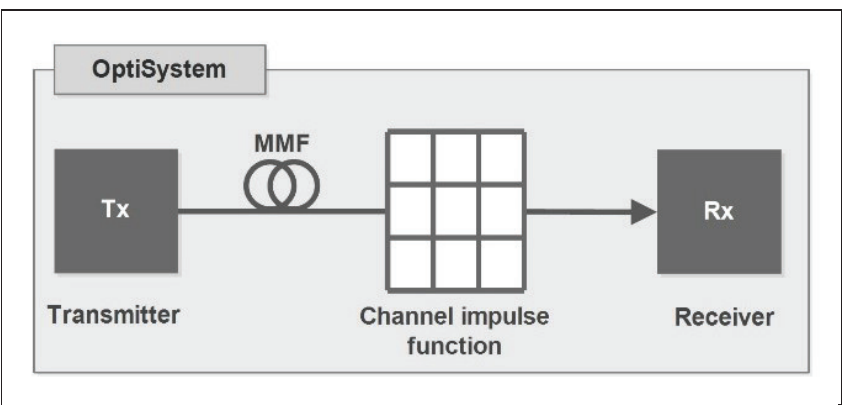

Fig. 1. MDM system model

MDM model consists of spatial optical transmitter (Tx) which includes optical source or transmitter and a transverse mode generator. The type of mode that have been used in this experiment is Laguerre Gaussian (LG). LG modes are extensions of the simple Gaussian mode shape to higher order modes in a cylindrical coordinate system, and are often used to describe the transverse mode shapes at the output of a VCSEL. The analytical representation of the mode $L_{\mathrm{ml}}$ is given by equation (1)[21].

$$
\begin{aligned}
\psi_{m l}(r, \phi)= & \alpha \cdot\left(\frac{2 r^{2}}{w_{0}^{2}}\right)^{\frac{L}{2}} \cdot L_{m}^{l}\left(\frac{2 r^{2}}{w_{0}^{2}}\right) \cdot \exp \left(-\frac{r^{2}}{w_{0}^{2}}\right) \\
& \exp \left(j \frac{\pi r^{2}}{\lambda R_{o}}\right) \cdot\left\{\begin{array}{l}
\cos (L \phi), l>=0 \\
\sin (L \phi), l<0
\end{array}\right\}
\end{aligned}
$$

where $\alpha$ is a normalization constant, $L=|1|, \lambda$ is the field wavelength, and is a generalized Laguerre polynomial. At the beam waist, the inverse of $R_{o}$ is zero, indicating a flat phase front. At any distance to the left or right of the waist the beam begins to diverge and $\mathrm{R}_{\mathrm{o}}$ becomes finite.

The resulted optical signal is transmitted through MMF component to the receiver $(\mathrm{Rx})$. In this study, a MMF with a core diameter of $62.5 \mu \mathrm{m}$ has been used to guide 2 spatial LG modes, namely, LG01, LG11 at 1550nm. The modulation type used in this experiment is NRZ. Table 1 summarize the parameters of MDM system model.
Table 1. Parameters of MDM system model

\begin{tabular}{|l|c|}
\hline \multicolumn{1}{|c|}{ Parameter } & value \\
\hline MMF length & $2 \mathrm{~km}$ \\
\hline Wavelength & $1550 \mathrm{~nm}$ \\
\hline Bit rate & $40 \mathrm{~GB} / \mathrm{s}$ \\
\hline modulation type & $\mathrm{NRZ}$ \\
\hline Mode type & $\mathrm{LG}$ \\
\hline Core & 62.5 \\
\hline
\end{tabular}

In the receiver, the photodetector is used to convert the optical signal into the electrical current. The output of MDM system model, which represents a signal with mode coupling effects, is used as an input signal to be recovered by using PSO equalization scheme. Next section will discuss in details the PSO equalization scheme.

\subsection{Modelling PSO equalization scheme}

In this study, the PSO equalization scheme has been proposed to improve the performance of MDM system by improving the mitigation of mode coupling and increase a bandwidth. The proposed scheme has been implemented at the receiver side for post-compensation, using Matlab platform. Figure 2 illustrates the PSO equalization scheme.

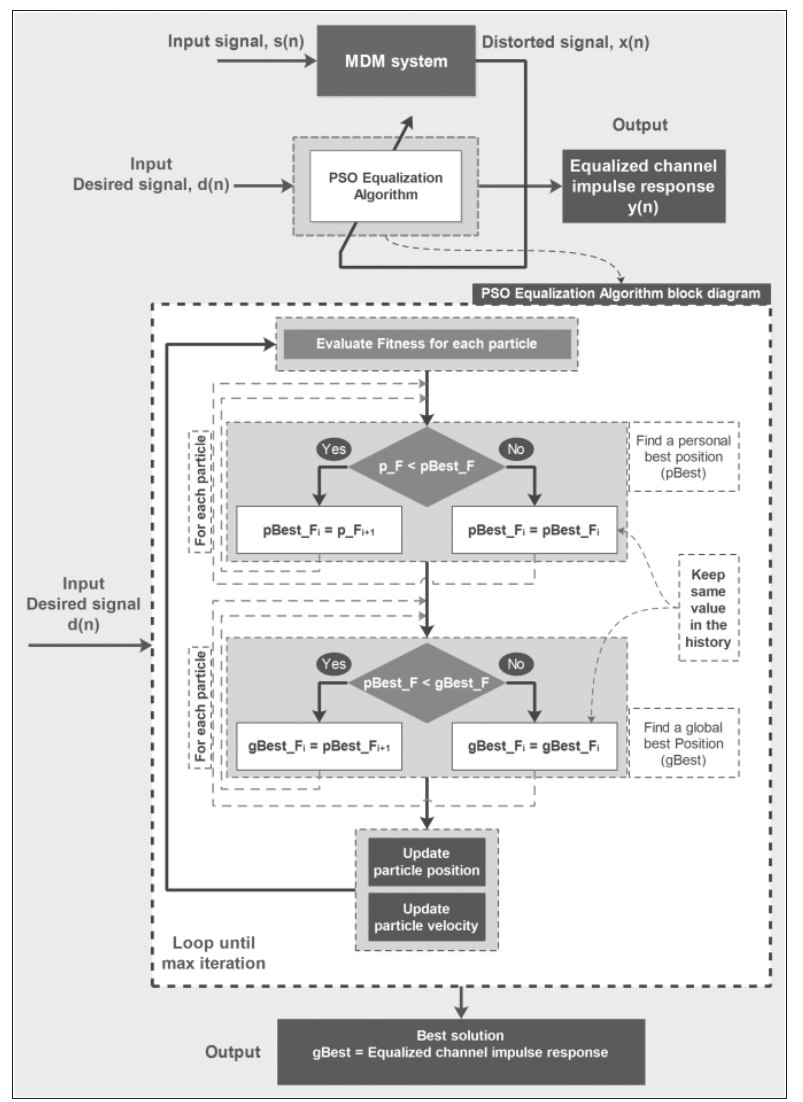

Fig. 2. PSO equalization scheme

The input of MDM system at time $n$, is the channel impulse function, $\mathrm{s}(\mathrm{n})$. The output of MDM system model, $\mathrm{x}(\mathrm{n})$ which represents a signal with mode coupling effects, termed as the channel impulse response in the time domain. The desired signal, $d(n)$ is assumed 
to be a Gaussian pulse, which is a standard ideal pulse shape[22]. The Gaussian pulse is shaped with mean $=$ 5.5 , and variance $=2.0, \mathrm{~d}(\mathrm{n})$ and $\mathrm{x}(\mathrm{n})$ are the inputs to the PSO equalization scheme.

The block diagram of PSO equalization scheme consists of: the evaluation fitness block to evaluate all particles (potential solutions). In order to find the particle with the best fitness value (pBest_F) in each iteration, which is called personal best position (pBest), the current fitness value $\left(\mathrm{p} \_\mathrm{Fi}+1\right)$ will be compared with the (pBest Fi) in the history. If $\mathrm{p}_{-} \mathrm{Fi}+1<\mathrm{pBest}$ _Fi then pBest_Fi $=$ p_Fi+1. Furthermore, to find the particles with the best fitness value between all the particles (gBest_F), known as global best position (gBest), the current fitness value (pBest_Fi) value, will compared with the (gBest_Fi) in the history. If pBest_Fi $<$ gBest_Fi +1 then gBest_Fi $=$ pBest Fi+1. The particle velocity and position will be updated by using (2) and (3) respectively.

$$
\begin{aligned}
& v_{i}(t+1)=\omega \cdot v_{i}(t)+c_{1} \cdot r_{1}\left(\text { pbest }_{i}(t)-x_{i}(t)\right) \\
& +c_{2} \cdot r_{2}\left(\operatorname{gbest}(t)-x_{i}(t)\right) \\
& x_{i}(t+1)=x_{i}(t)+v_{i}(t+1)
\end{aligned}
$$

where $\mathrm{c} 1$ is cognitive parameter and $\mathrm{c} 2$ is a social parameter that propel solutions toward personal best and global best respectively; $\mathrm{r} 1$ and $\mathrm{r} 2$ are random numbers with uniform distribution, $\omega$ is an inertia weight (w) used to control the velocity. The output of PSO equalization algorithm (gBest) is the equalized channel impulse response, becomes as close as possible to a desired signal d(n). Table 2 summarize the parameters' values of PSO algorithm.

Table 2. Parameters' values of PSO algorithm

\begin{tabular}{|l|c|}
\hline Parameter & Value \\
\hline $\mathrm{c}_{1}$ & 2.0 \\
\hline $\mathrm{c}_{2}$ & 1.5 \\
\hline $\mathrm{w}_{0}$ & 1.0 \\
\hline $\mathrm{w}$ & 0.98 \\
\hline Max iteration & 50 \\
\hline Swarm size & 20 \\
\hline Terminal value & $<=0.00001$ \\
\hline
\end{tabular}

\section{Result}

The performance of PSO equalization scheme was compared with equalization based on LMS and RLS algorithms. Two channels were used as a distorted signal, similarly, two Gaussian signals were used as a desired signal. The mean square error (MSE) has been used to measure the differences between desired signal and distorted signal, before equalization and between desired signal and equalized impulse response after equalization. Figure 3 shows the MSE before and after equalization using LMS, RLS and PSO algorithm. From the results it can be clearly seen that the PSO algorithm outperforms LMS and RLS algorithms with less MSE values for both channel 1 and channel 2. Figure 4 shows the differences between impulse responses of channel 1 and channel2, before and after performing PSO equalization scheme. Before the equalization, the symbols of channel 1 and channel 2 have been overlapped with each other, while after PSO equalization the impulse responses of channel 1 and channel 2 have been recovered as shown in Figure 5.

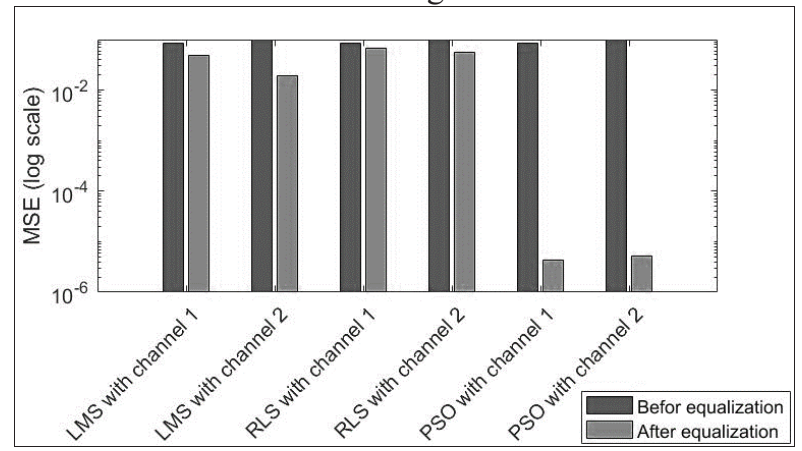

Fig. 3. MSE before and after equalization using LMS, RLS and PSO algorithms

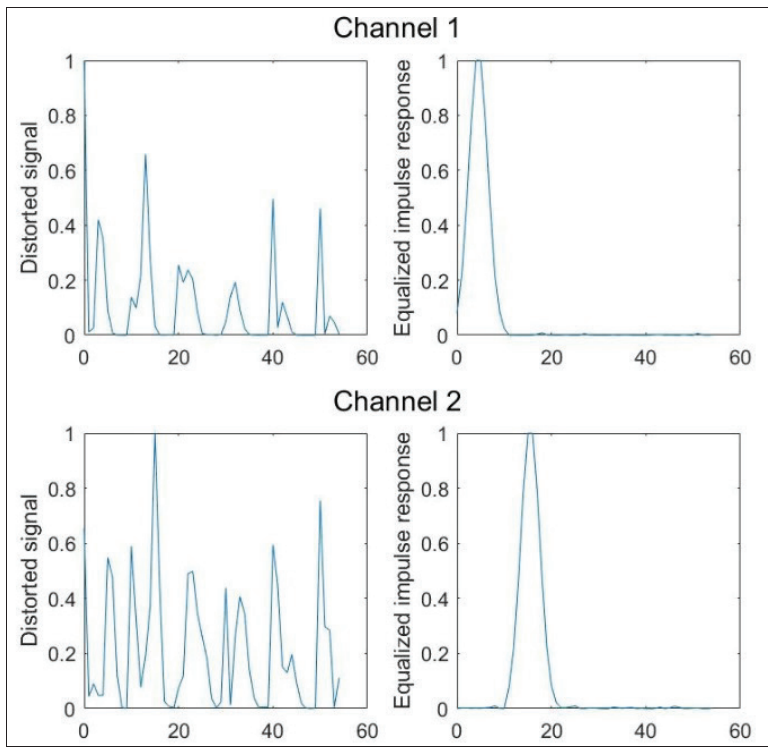

Fig. 4. Channel 1 and Channel 2 before and after PSO equalization scheme

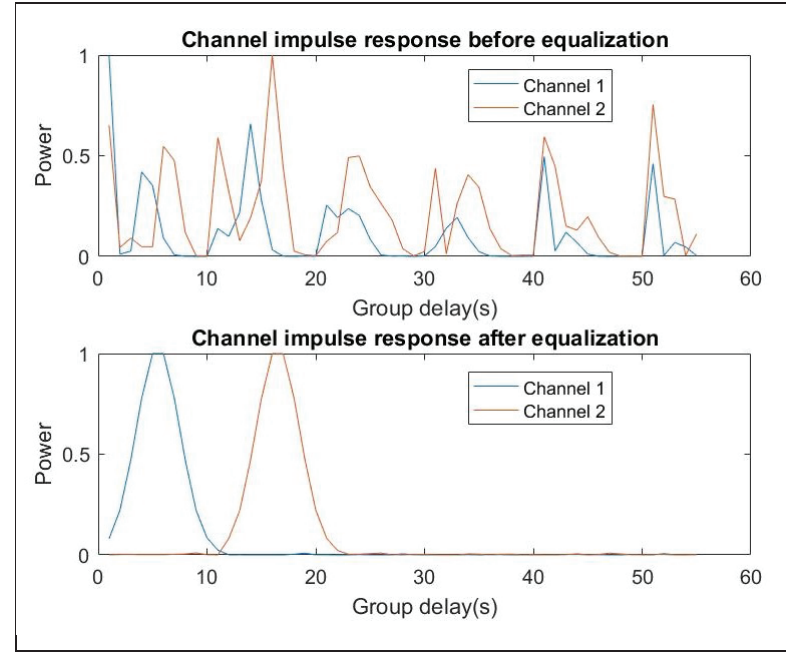

Fig. 5. Channel impulse response before and after PSO equalization scheme 


\section{Conclusion}

An PSO equalization scheme is proposed and simulated for MDM system with MMF of length $2 \mathrm{~km}$. It is shown that the proposed PSO equalization scheme significantly reduces the mode coupling effects compared to LMS and RLS algorithms.

\section{References}

1. Cisco, CISCO White paper, (2015)

2. B. Batagelj, V. Janyani, and S. Tomažič, Informacije MIDEM, 44, no. 3, pp. 177-184 (2015)

3. B. Woodward and E. B. Husson, Fiber optics installer and technician guide. (John Wiley \& Sons, 2006)

4. S. O. Arik, J. M. Kahn, and K. P. Ho, IEEE Signal Processing Magazine, 31, no. 2, pp. 2534 (2014)

5. D. Richardson, J. Fini, and L. Nelson, Nature Photonics, 7, no. 5, pp. 354-362 (2013)

6. N. Bai and G. Li, Optics express, 22, no. 4, pp. 4247-4255 (2014)

7. A. Amphawan and N. M. A. Al Samman, SPIE Optical Engineering \& Applications, 8847, pp. 88470Y-88470Y-6 (2013)

8. T. Masunda and A. Amphawan, Journal of Optical Communications (2016)

9. C. Yue, X. Kewen, and L. Jianfei, Advances in Information Sciences and Service Sciences, 5, no. 9, p. 114 (2013)

10. N. Iqbal, A. Zerguine, and N. Al-Dhahir, Signal Processing, 108, pp. 1-12 (2015)

11. X. Xiang et al., 2015 14th International Conference on Optical Communications and Networks (ICOCN), pp. 1-3 (2015)

12. R. Ryf et al., Optical Fiber Communication Conference and Exposition (OFC/NFOEC), 2011 and the National Fiber Optic Engineers Conference pp. 1-3 (2011)

13. C. Koebele et al., European Conference and Exposition on Optical Communications (2011)

14. S. A. A. Khan and S. A. Sheikh, The Second International Conference on Electrical, Electronics, Computer Engineering and their Applications (EECEA2015) p. 163 (2015)

15. L. Yan and G. Hu, IEEE Photonics Journal, 8, no. 2, pp. 1-11 (2016)

16. N. Bai and G. Li, IEEE Photonics Technology Letters, 24, no. 21, pp. 1918-1921 (2012)

17. Y. Weng, X. He, and Z. Pan, SPIE OPTO, pp. 97740B-97740B-12 (2016)

18. S. Yogi, K. Subhashini, J. Satapathy, and S. Kumar, 2010 IEEE International Conference on Communication Control and Computing Technologies (ICCCCT), pp. 725-730 (2010)

19. N. Sharma, A. K. Tarcar, V. A. Thomas, and K. Anupama, Information Sciences, 182, no. 1, pp. 115-124 (2012)
20. A. Amphawan, Y. Fazea, and M. Elshaikh, Advanced Computer and Communication Engineering Technology: Proceedings of ICOCOE (2015)

21. H. A. Sulaiman, M. A. Othman, M. F. I. Othman, Y. A. Rahim, and N. C. Pee, Eds. Cham: Springer International Publishing $\mathrm{pp}$. 355-363 (2016)

22. C. E. Webb and J. D. Jones, Handbook of Laser Technology and Applications: Laser design and laser systems. (CRC Press, 2004) 